\title{
Papers
}

\section{Randomised controlled trial assessing the impact of a nurse delivered, flow monitored protocol for optimisation of circulatory status after cardiac surgery}

\author{
Moira McKendry, Helen McGloin, Debbie Saberi, Libby Caudwell, Anthony R Brady, Mervyn Singer
}

\begin{abstract}
Objective To assess whether a nurse led, flow monitored protocol for optimising circulatory status in patients after cardiac surgery reduces complications and shortens stay in intensive care and hospital.

Design Randomised controlled trial.

Setting Intensive care unit and cardiothoracic unit of a university teaching hospital.

Participants 174 patients who underwent cardiac surgery between April 2000 and January 2003.

Interventions Patients were allocated to conventional haemodynamic management or to an algorithm guided by oesophageal Doppler flowmetry to maintain a stroke index above $35 \mathrm{ml} / \mathrm{m}^{2}$.

Results 26 control patients had postoperative complications (two deaths) compared with 17 (four deaths) protocol patients $(\mathrm{P}=0.08)$. Duration of hospital stay in the protocol group was significantly reduced from a median of nine (interquartile range $7-12)$ days to seven (7-10) days $(\mathrm{P}=0.02)$. The mean duration of hospital stay was reduced from 13.9 to 11.4 days, a saving in hospital bed days of $18 \%$ (95\% confidence interval - $12 \%$ to $47 \%)$. Usage of intensive care beds was reduced by $23 \%(-8 \%$ to $59 \%)$.

Conclusion A nurse delivered protocol for optimising circulatory status in the early postoperative period after cardiac surgery may significantly shorten hospital stay.
\end{abstract}

\section{Introduction}

Hypovolaemia and tissue hypoperfusion can pass undetected during and after major surgery. ${ }^{1}$ The resulting systemic inflammatory response and organ dysfunction, often not clinically apparent for several days, may lead to increased morbidity and mortality and prolonged hospital stay.

Several perioperative studies have used invasive (pulmonary artery catheterisation) or minimally invasive (oesophageal Doppler flowmetry) monitoring technologies to optimise circulatory variables such as stroke volume, cardiac output, arterial lactate concentration, or oxygen delivery and consumption. ${ }^{2}$ Although a similar approach proved unsuccessful in improving outcomes in critically ill patients with organ failure, these studies showed major improvements in postoperative complications and stay in intensive care or hospital. By comparison, a recent Canadian study found no improvement in outcomes in patients allocated to pulmonary artery catheterisation but in whom a protocol for haemodynamic optimisation was not followed perioperatively. ${ }^{3}$
To our knowledge, only one randomised study (403 patients) has specifically investigated optimisation of circulatory status after cardiac surgery. ${ }^{4}$ This study reported a reduction in median duration of hospital stay from seven to six days $(\mathrm{P}<0.05)$ in patients targeted to achieve a mixed venous saturation in excess of $70 \%$ and a plasma lactate concentration below $2 \mathrm{mmol} / \mathrm{l}$ in the first eight hours postoperatively. We previously reported that a low stroke volume index $\left(<35 \mathrm{ml} / \mathrm{m}^{2}\right)$ and a high heart rate on admission to intensive care after cardiac surgery and at four hours were the best prognostic factors for the development of subsequent complications. ${ }^{5}$ We therefore studied the optimisation of circulatory status in patients in the first four hours after admission to intensive care after cardiac surgery. Half the patients were randomised to receive treatment (fluid with or without vasodilators and inotropes) guided by oesophageal Doppler flowmetry to achieve a stroke volume index above 35 $\mathrm{ml} / \mathrm{m}^{2}$. This trial differs from the previous study in two major respects. ${ }^{4}$ Firstly, cardiac output was monitored using minimally invasive technology (oesophageal Doppler flowmetry) and, secondly, nurses conducted the study using a protocol driven approach with minimal additional input from medical staff.

\section{Participants and methods}

Our study was conducted on the intensive care and postoperative cardiothoracic units of the University College London Hospitals NHS Trust. Enrolment was performed by any one of the clinical study investigators. Participants were patients undergoing cardiopulmonary bypass surgery who had provided informed consent preoperatively. We excluded patients undergoing off-pump surgery, those who declined to consent, those aged under 18 years, or those with relative contraindications to the use of the oesophageal Doppler probe, such as oesophageal varices or other oropharyngeal and oesophageal disease. Patients were also excluded postoperatively if on admission to intensive care there was excessive bleeding, unstable arrhythmias, a need for intra-aortic balloon counterpulsation, or inotrope requirements $\geq 10 \mu \mathrm{g} / \mathrm{kg} / \mathrm{min}$ of dopamine or dobutamine or $\geq 0.16$ $\mu \mathrm{g} / \mathrm{kg} / \mathrm{min}$ of adrenaline (epinephrine) or noradrenaline (norepinephrine).

For the oesophageal Doppler technique a $6 \mathrm{~mm}$ probe was placed into the lower oesophagus through the mouth or nose. The probe is directed to detect midstream blood flow in the descending thoracic aorta, with the aid of an online monitor (to obtain sharply defined flow velocity waveforms with minimal spectral dispersion) and an integral loudspeaker (to detect maximal pitch). 


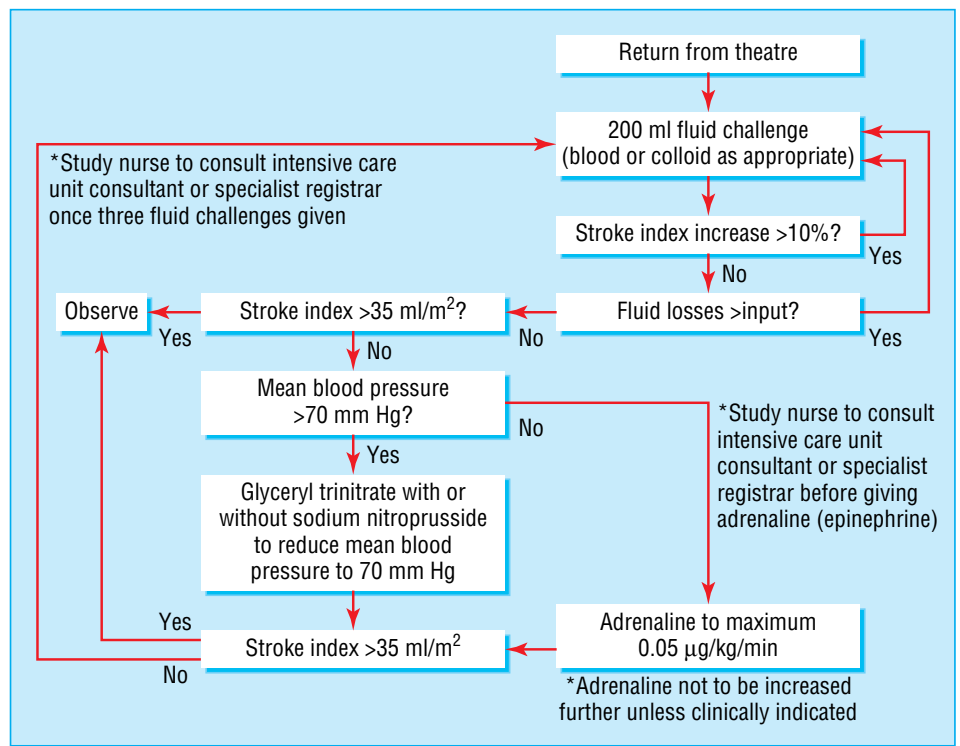

Fig 1 Treatment algorithm to optimise circulatory status in patients after cardiac surgery

\section{Objectives and intervention}

Our primary objective was to compare the lengths of stay in intensive care and hospital after cardiac surgery in patients receiving standard perioperative care or optimisation of circulatory status in the first four hours postoperatively. Our secondary objective was to compare postoperative complications between the two groups.

\section{Control group}

Standard postoperative care in our institution, as in most UK cardiac surgical centres, does not involve monitoring cardiac output but relies primarily on monitoring arterial and central venous pressures with markers of tissue perfusion such as urine output and arterial base deficit. The control group thus received conventional management as determined by the intensive care and cardiac surgical teams. This could include monitoring of cardiac output if considered clinically indicated. The study nurse took readings from these patients with an oesophageal Doppler probe (CardioQ, Deltex, Chichester, Sussex) within 10 minutes of admission to the intensive care unit and at four hours postoperatively. Doctors and nurses not involved with the study were not allowed sight of these readings, and the machine was switched off in the interim. If patients in either group were ready for extubation before four hours, a Doppler recording was made before removal of the endotracheal tube.

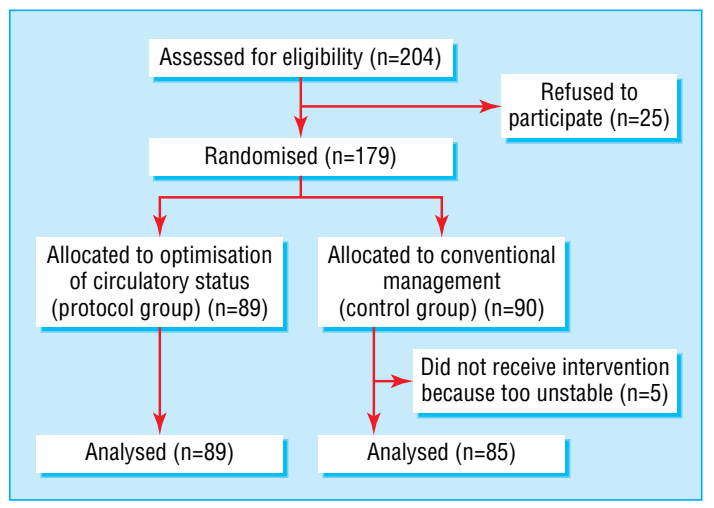

Fig 2 Flow of participants through trial

\section{Protocol group}

The oesophageal Doppler probe was also inserted within 10 minutes of arrival of the protocol patients on the intensive care unit. The algorithm in figure 1 was followed to increase the stroke volume index to $\geq 35 \mathrm{ml} / \mathrm{m}^{2}$ or greater using repeated colloid challenges, with nitrates and inotropes given as required. This management was directed by the study nurse, but with clear indications for referral to medical staff.

Recordings of standard haemodynamic variables and intravenous fluid and drug requirements were made manually over the first four hours. Follow up data were collected by the study nurses on days 1,2 , and 5 postoperatively, including complications, time to extubation, and length of stay in intensive care and hospital. Data were entered in a database and cross checked for accuracy. If the patient was medically fit for hospital discharge but this was delayed for social or logistical reasons, a note was made. Both patients and staff on the general wards to which patients were sent after discharge from intensive care were unaware of the group assignment.

Table 1 Baseline characteristics of patients randomised after cardiac surgery to conventional management or protocol for optimising cardiac variables. Values are numbers (percentages) unless stated otherwise

\begin{tabular}{lcc} 
Characteristics & $\begin{array}{c}\text { Control group } \\
(\mathbf{n}=\mathbf{8 5})\end{array}$ & $\begin{array}{c}\text { Protocol group } \\
(\mathbf{n = 8 9})\end{array}$ \\
\hline Mean (SD) age (years) & $66.7(10.2)$ & $65.6(10.3)$ \\
\hline Male & $57(67)$ & $56(63)$ \\
\hline Mean (SD) Parsonnet score ${ }^{*}$ & $9.7(7.6)$ & $9.7(7.5)$ \\
\hline Diabetes mellitus & $19(22)$ & $16(18)$ \\
\hline Previous myocardial infarction & $21(25)$ & $23(26)$ \\
\hline History of hypertension & $30(35)$ & $33(37)$ \\
\hline $\begin{array}{l}\text { Taking angiotensin converting enzyme inhibitor or } \\
\text { diuretic }\end{array}$ & $30(35)$ & $28(31)$ \\
\hline Elective surgery & $71(84)$ & $78(88)$ \\
\hline Type of surgery: & $51(60)$ & $53(60)$ \\
\hline Coronary artery bypass graft & $2(2)$ & $2(2)$ \\
\hline Repeat coronary artery bypass graft & $22(26)$ & $23(26)$ \\
\hline Valve replacement & $10(12)$ & $9(10)$ \\
\hline Coronary artery bypass graft and valve replacement & 0 & $2(2)$ \\
\hline Other & &
\end{tabular}

*Risk related to cardiac surgery: 0-4 (good) to $\geq 20$ (extremely high) 
Table 2 Management of patients in four hours after cardiac surgery. Values are means (standard deviations)

\begin{tabular}{lccc} 
Measure & $\begin{array}{c}\text { Control group } \\
(\mathbf{n = 8 5})\end{array}$ & $\begin{array}{c}\text { Protocol group } \\
(\mathbf{n}=\mathbf{8 9})\end{array}$ & P value \\
\hline Fluid requirement: & & & \\
\hline Colloid $(\mathrm{ml})$ & $1042(620)$ & $1667(464)$ & $<0.001$ \\
\hline Crystalloid $(\mathrm{ml})$ & $328(99)$ & $353(95)$ & 0.09 \\
\hline Change in measure: & & & \\
\hline Stroke volume $(\mathrm{ml})$ & $-10.2(18.4)$ & $1.5(18.9)$ & $<0.001$ \\
\hline Cardiac output $(\mathrm{l} / \mathrm{min})$ & $-0.46(1.68)$ & $0.45(1.98)$ & 0.001 \\
\hline Corrected flow volume (ms) & $-32.5(59.7)$ & $-6.2(67.5)$ & 0.007 \\
\hline Peak velocity (cm/s) & $-2.7(16.6)$ & $2.6(14.4)$ & 0.03 \\
\hline Arterial base excess (mmol/l) & $-0.73(1.60)$ & $-0.54(2.19)$ & 0.50 \\
\hline
\end{tabular}

\section{Randomisation and sample size calculation}

Patients were randomised by an a priori computer generated sequence. The study nurse opened the serially numbered, sealed, opaque envelopes on arrival of patients at intensive care, provided exclusion criteria were not met.

We calculated a sample size requirement of 170 patients $(85$ in each group) by postulating a reduction in mean hospital stay from 13 days in the control group to 10 days in the protocol group $(\alpha=0.05$, power 0.9$)$. Differences in postoperative measurements were tested using two sided $t$ tests when normal plots indicated this was appropriate. We used the two sample Wilcoxon rank sum test to test for differences in non-normally distributed variables, including the main outcomes (length of stay in intensive care and hospital). Confidence intervals were constructed for the difference in mean length of stay between the two groups by bootstrapping within treatment groups $(10000$ replications). ${ }^{6}$ The analyses were performed on an intention to treat basis. We also investigated the effect of excluding from these analyses patients who died in hospital. Pearson's $\chi^{2}$ test was used to compare complications between treatment groups. Analyses were carried out with Stata (release 7.0) software.

\section{Results}

Overall, 179 patients were recruited between April 2000 and January 2003 (fig 2). Five patients who provided consent before surgery did not fulfil the postoperative entry criteria. Four of these five patients were erroneously randomised but are not included in subsequent analyses, leaving 89 patients in the protocol group and 85 in the control group. The groups were well matched for age, sex, weight, Parsonnet cardiac risk score, and type of surgery (table 1). ${ }^{7}$ The median first 24 hour acute physiological and chronic health evaluation II score was similar in both groups (10 protocol, 11 control). These patients are representative of the total cardiac surgical population of the hospital, whose Parsonnet score averaged 9.0 (SD 7.4), with a similar frequency of coronary artery bypass grafting with or without valve replacement.

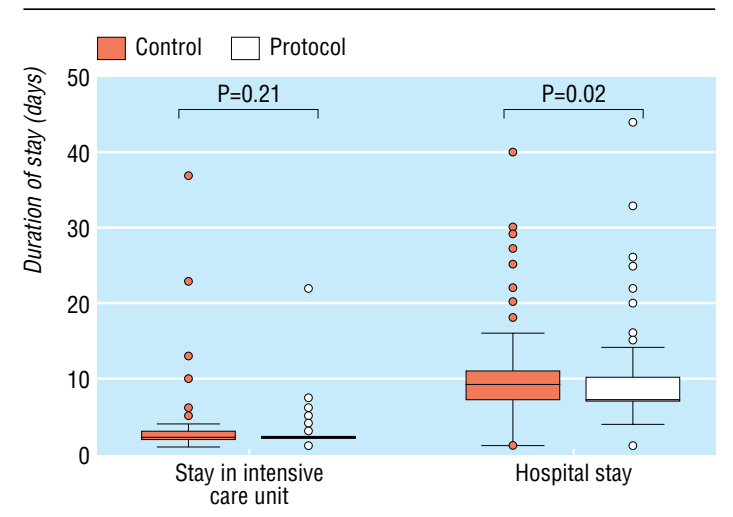

Fig 3 Duration of stay in intensive care and hospital in patients receiving strategy to optimise circulatory status after cardiac surgery (protocol) or conventional management (control). Six hospital stays beyond 50 days not shown

Table 2 shows the haemodynamic data and fluid requirements over the initial four hour postoperative period. Although stroke volume index, cardiac index, and use of colloid were well matched at baseline (within 10 minutes of admission), they were significantly greater in the protocol group at four hours; use of inotropes was similar between the two groups. Colloid was given to all but one of the control patients. None of the control patients had pulmonary artery catheters in situ or had Doppler probes placed because of perceived clinical need. Inotropes were not instituted as per protocol to increase stroke volume index values to $35 \mathrm{ml} / \mathrm{m}^{2}$ or greater owing to the short duration of the study and the frequent need for repeated fluid challenges in the study period. At four hours, 35 (39\%) protocol patients and 48 (56\%) control patients had values below $35 \mathrm{ml} / \mathrm{m}^{2}$.

In the protocol group, the mean number of days in intensive care, although not statistically significant, was reduced from 3.2 to 2.5 (23\% reduction, $95 \%$ confidence interval $-8 \%$ to $59 \%$; fig 3 ). The mean duration of hospital stay in this group was reduced from 13.9 to 11.4 days (18\% reduction, $-12 \%$ to $47 \%$ ), with a significant reduction in median duration of stay from nine to seven days $(\mathrm{P}=0.02)$. Delayed hospital discharge for nonmedical reasons affected four protocol patients (total delay 23 days) and one control patient (three days).

Four deaths occurred in the protocol group and two in the control group, the causes of which were not considered directly attributable to early postoperative care (table 3). Comparisons of length of stay in survivors only were similar to those for all patients. The protocol group showed a trend towards fewer major postoperative complications and deaths than the control group (table 4).

\section{Table 3 Details of deaths after cardiac surgery}

\begin{tabular}{lllccll}
$\begin{array}{l}\text { Age } \\
\text { (years) }\end{array}$ & Sex & $\begin{array}{c}\text { Randomisation } \\
\text { group }\end{array}$ & $\begin{array}{c}\text { Parsonnet } \\
\text { score }^{*}\end{array}$ & $\begin{array}{c}\text { Postoperative } \\
\text { day of death }\end{array}$ & Type of operation \\
\hline 80 & Male & Protocol & 20 & 1 & Repeat coronary artery bypass graft & Cause of death \\
\hline 75 & Male & Protocol & 14 & 4 & Repeat coronary artery bypass graft & Lleeding, cardiac arrest (pulseless electrical activity) \\
\hline 73 & Male & Protocol & 17 & 7 & Aortic valve replacement & $\begin{array}{l}\text { Grand mal seizures, low blood pressure, multiple organ } \\
\text { failure }\end{array}$ \\
\hline 68 & Female & Protocol & 8 & 22 & Mitral valve replacement and coronary artery bypass graft & Anterior myocardial infarction on day 3; multiple organ failure \\
\hline 83 & Male & Control & 25 & 1 & Aortic valve replacement & Massive bleeding \\
\hline 76 & Female & Control & 10 & 10 & Aortic valve replacement & Anterior myocardial infarction on day 2; multiple organ failure
\end{tabular}

${ }^{*}$ Risk related to cardiac surgery: 0-4 (good) to $\geq 20$ (extremely high). 
Table 4 Number of patients with complications after cardiac surgery

\begin{tabular}{lcc} 
Postoperative complication & $\begin{array}{c}\text { Protocol } \\
\text { group }\end{array}$ & $\begin{array}{c}\text { Control } \\
\text { group }\end{array}$ \\
\hline Atrial fibrillation requiring treatment & 5 & 11 \\
\hline Pneumothorax & 1 & 2 \\
\hline Cerebral vascular accident & 1 & 2 \\
\hline Chest infection or sternal wound infection & 1 & 4 \\
\hline Gastrointestinal bleed or perforated duodenal ulcer & 1 & 2 \\
\hline Acute renal failure & 1 & 3 \\
\hline Pleural effusion & 2 & 1 \\
\hline Infected leg wound & 1 & 1 \\
\hline Aortic regurgitation & 0 & 1 \\
\hline Death & 4 & 2 \\
\hline Any of above & 17 & 26
\end{tabular}

\section{Discussion}

A nurse delivered protocol to optimise circulatory status of patients early after cardiac surgery, using oesophageal Doppler flowmetry and targeted at improving stroke volume, reduced the length of hospital stay. This protocol was also associated with a trend towards fewer complications and reduced stay in intensive care.

Tissue hypoperfusion is common in patients perioperatively and is associated with high postoperative morbidity and mortality. ${ }^{8-11}$ One study found that the magnitude of the tissue organ debt perioperatively was related to the subsequent development of organ failure and death. ${ }^{11}$ Tissue hypoxia is a potent stimulus for an inflammatory response. It may synergise with coexisting infection or endotoxaemia to produce an amplified systemic inflammatory response that may progress to multiple organ dysfunction. A low mortality $(3.6 \%)$ was reported in a series of 2609 consecutive patients after cardiac surgery, yet over one third of the patients had one or more postoperative complications, with non-cardiac complications being associated with more adverse outcomes. ${ }^{12}$ As in our study, these were independent of Parsonnet score and bypass time.

Optimisation of circulatory status perioperatively was a concept first promulgated by Shoemaker et al. ${ }^{13}$ They found a significant reduction in mortality and hospital stay in high risk surgical patients receiving fluid loading with or without dobutamine to increase cardiac output, delivery of oxygen to tissues, and oxygen consumption to "supranormal" values. Comparable results from other groups using a similar goal directed approach lends further support to the importance of avoiding a tissue oxygen debt perioperatively. ${ }^{11}{ }^{14}$ In large Canadian and US studies, however, placement of a pulmonary artery catheter without targeting predefined haemodynamic end points produced no difference in postoperative outcomes. ${ }^{3}{ }^{16}$ In one of the studies there was no significant difference in the volume of fluid given to the two treatment groups (J D Sandham, personal communication, 2003). These studies support the generalisability of our results to countries where monitoring of cardiac output is performed more often during cardiac surgery than in the United Kingdom, and, importantly, also emphasise the need to obtain maximum utility from a monitoring technique.

The necessity for treatment to be directed by invasive monitoring (pulmonary artery catheterisation) has been challenged by several groups (including ourselves) that used oesophageal Doppler flowmetry to maximise intraoperative stroke volume by repeated fluid challenges. ${ }^{17-20}$ These studies showed important $(30-40 \%)$ reductions in length of hospital stay after cardiac, orthopaedic, or abdominal surgery. The lesser reduction in mean length of hospital stay $(18 \%)$ achieved by us is comparable to that
What is already known on this topic

After cardiac surgery many patients have complications that prolong hospital stay

Perioperative tissue hypoxia is the postulated trigger for many of these complications

What this study adds

Optimisation of intravascular volume in the first four hours postoperatively reduces complications and bed usage

Nurses could deliver the protocol using a minimally invasive device that monitors stroke volume and responses to interventions

reported by Polonen et al. ${ }^{4}$ These data imply that optimisation of circulatory status should ideally begin at the start of, if not before, a major operation, but that some advantage is still gained from optimisation immediately after surgery.

As shown by our study, and by standard practice on many UK intensive care units, nurses can safely insert and align the oesophageal Doppler probe to detect descending aortic blood flow within a few minutes, thus obviating the need for help from clinicians. The size of the waveform displayed on the monitor reflects stroke volume (a reasonable estimate of total left ventricular output is achieved by an integral nomogram), whereas the waveform shape provides other useful information on left ventricular contractility, preload and afterload. ${ }^{21-23}$ Although staff could not be blinded to the randomisation schedule, the subsequent management of patients after the first four hours was identical and they were not made aware of the Doppler results. Complications were objectively recorded by hard end points such as the need for antibiotics and antiarrhythmics.

The intention to use inotropes if fluid loading failed to achieve a target stroke index was not met, mainly because of time constraints but in part to the reticence of the nurses. Our study should thus be viewed as a trial of immediate optimisation of fluid status in the postoperative period. Whether a subset of the patients would benefit from a more aggressive intervention, including inotropes, or a presurgical exercise regimen to increase their anaerobic threshold ${ }^{24}$ requires further investigation.

The weaknesses of our trial were that it was of relatively small size and conducted in only one centre. A larger multicentre trial is needed to confirm the generalisability of our findings and the effect on mean length of hospital stay, which is more relevant to costs than median duration of stay. ${ }^{6}$ The disparity between the mean and median results in our study highlights the fact that although many patients who are conventionally managed after cardiac surgery do not develop clinically apparent morbidity, a greater proportion will develop complications requiring additional utilisation of resources postoperatively. For example, 13 control patients required four or more days of intensive care compared with six protocol patients.

In conclusion, improvements in postoperative outcomes in patients after cardiac surgery may be achieved by early intervention targeted at optimising the stroke volume. A nurse delivered, protocol driven approach has the potential for widespread application as it is not routinely feasible for a clinician to be constantly at the bedside. 
We thank for their cooperation the nursing and medical staff at the Heart Hospital and previously at the Middlesex Hospital intensive care and postoperative cardiothoracic units.

Contributors: MS had the original idea for the study. MS, LC, DS, and $\mathrm{HMcG}$ designed the protocol. MMcK, LC, DS, and HMcG performed the bedside study. MS, MMcK, and ARB analysed the data and wrote the manuscript. MS will act as guarantor for the paper.

Funding: Deltex provided an unrestricted educational grant for this study. Competing interests: Deltex provided research funds to the department and contributed towards the salaries of the research nurses (LC, DS, MMcK, and $\mathrm{HMcG}$ ) and travel expenses and registration fees to enable them to present their research at national and international congresses. MS does consultancy work for Deltex.

Ethical approval: Ethics committee of the University College London Hospitals NHS Trust.

1 Shoemaker WC, Wo CC, Thangathurai D, Velmahos G, Belzberg H, Asensio JA, et al. Haemodynamic patterns of survivors and non survivors during high risk elective surgical operations. World J Surg 1999;23:1264-70.

2 Kern JW, Shoemaker WC. Meta analysis of hemodynamic optimisation in high risk patients. Crit Care Med 2002;30:1686-9.

3 Sandham JD, Hull RD, Brant RF, Knox L, Pineo GF, Doig CJ, et al. A randomised, controlled trial of the use of pulmonary artery catheters in high risk surgical patients. $N$ Engl J Med 2003;348:5-14.

4 Polonen P, Ruokonen E, Hippelainem M, Poyhonen M, Takala J. A prospective randomised study of goal oriented hemodynamic therapy in cardiac surgical patients.
rants Anaesth Analg 2000:90:1052-9.

5 Poeze M, Ramsay G, Greve JWM, Singer M. Prediction of post operative cardiac surgical morbidity and organ failure within 4 hours of ICU admission using esophageal Doppler ultrasonography. Crit Care Med 1999;27:1288-94.

6 Thompson SG, Barber JA. How should cost data in pragmatic randomised trials be analysed? BMJ 2000;320:1197-200.

7 Parsonnet V, Dean D, Bernstein AD. A method of uniform stratification of risk for evaluating the results of surgery in acquired adult heart disease. Circulation 1989;79:I312.

8 Mythen MG, Webb AR. Intra-operative gut mucosal hypoperfusion is associated with increased post-operative complications and cost. Intensive Care Med 1994;20:99-104.

9 Shoemaker WC, Thangathurai D, Wo CC, Kuchta K, Canas M, Sullivan MJ, et al. Intraoperative evaluation of tissue perfusion in high-risk patients by invasive and noninvaoperative evaluation of tissue perfusion in high-risk patients by
sive hemodynamic monitoring. Crit Care Med 1999;27:2147-52.

10 Maillet JM, Le Besnerais P, Cantoni M, Nataf P, Ruffenach A, Lessana A, et al. Frequency, risk factors, and outcome of hyperlactatemia after cardiac surgery. Chest 2003;123:1361-6.

11 Shoemaker WC, Appel PL, Kram HB. Role of oxygen debt in the development of organ failure sepsis, and death in high-risk surgical patients. Chest 1992;102:208-15.

12 Welsby IJ, Bennett-Guerrero E, Atwell D, White WD, Newman MF, Smith PK, et al. The association of complication type with mortality and prolonged stay after cardiac surgery with cardiopulmonary bypass. Anesth Analg 2002;94:1072-8.
13 Shoemaker WC, Appel PL, Kram HB, Waxman K, Lee TS. Prospective trial of supranormal values of survivors as therapeutic goals in high-risk surgical patients. Chest 1988:94:1176-86.

14 Boyd O, Grounds RM, Bennett ED. A randomized clinical trial of the effect of deliberate perioperative increase of oxygen delivery on mortality in high-risk surgical patients. JAMA 1993;270:2699-707.

15 Wilson J, Woods I, Fawcett J, Whall R, Dibb W, Morris C, et al. Reducing the risk of major elective surgery: randomised controlled trial of preoperative optimisation of oxygen delivery. BMJ 1999;318:1099-103.

16 Polanczyk CA, Rohde LE, Goldman L, Cook EF, Thomas EJ, Marcantonio ER, et al Right heart catheterization and cardiac complications in patients undergoing noncardiac surgery: an observational study. JAMA 2001;286:309-14.

17 Mythen MG, Webb AR. Perioperative plasma volume expansion reduces the incidence of gut mucosal hypoperfusion during cardiac surgery. Arch Surg 1995;130:423-9.

18 Sinclair S, James S, Singer M. Intraoperative intravascular volume optimisation and length of hospital stay after repair of proximal femoral fracture: randomised controlled trial. BMJ 1997;315:909-12.

19 Gan TJ, Soppitt A, Maroof M, el-Moalem H, Robertson KM, Moretti E, et al. Goal-directed intraoperative fluid administration reduces length of hospital stay after major surgery. Anesthesiology 2002;97:820-6.

20 Venn R, Steele A, Richardson P, Poloniecki J, Grounds M, Newman P. Randomized controlled trial to investigate influence of the fluid challenge on duration of hospital stay and perioperative morbidity in patients with hip fractures. BrJ Anaesth 2002;88:65stay

21 Valtier B, Cholley BP, Belot JP, de la Coussaye JE, Mateo J, Payen DM. Noninvasive monitoring of cardiac output in critically ill patients using transesophageal Doppler. Am J Respir Crit Care Med 1998;158:77-83.

22 Singer M, Bennett ED. Noninvasive optimization of left ventricular filling using esophageal Doppler. Crit Care Med 1991;19:1132-7.

23 Singer M, Allen MJ, Webb AR, Bennett ED. Effects of alterations in left ventricular filling, contractility and systemic vascular resistance on the ascending aortic blood velocity waveform of normal subjects. Crit Care Med 1991;19:1138-45.

24 Older P, Smith R, Courtney P, Hone R. Preoperative evaluation of cardiac failure and ischemia in elderly patients by cardiopulmonary exercise testing. Chest 1993;104:701-4. (Accepted 24 May 2004)

doi $10.1136 /$ bmj.38156.767118.7C

Bloomsbury Institute of Intensive Care Medicine, Department of Medicine and Wolfson Institute of Biomedical Research, University College London, Middlesex Hospital, London W1T 3AA

Moira McKendry research sister

Helen McGloin research sister

Debbie Saberi research sister

Libby Caudwell research sister

Mervyn Singer professor of intensive care

Intensive Care National Audit and Research Centre, BMA House, London

WC1H 9JR

Anthony R Brady statistician

Correspondence to: M Singer m.singer@ucl.ac.uk 